\title{
Comparación de dos técnicas propuestas HS-CbCr y HS-ab para el modelado de color de piel en imágenes
}

\author{
Diana Alejandra Contreras Alejo, Francisco Javier Gallegos Funes \\ Instituto Politécnico Nacional, ESIME Zacatenco, \\ S.E.P.I. Doctorado en Comunicaciones y Electrónica, Cd. de México, \\ México \\ dianalecontreras@gmail.com, fgallegosf@ipn.mx
}

\begin{abstract}
Resumen. El contenido de este trabajo es la propuesta de dos técnicas para el modelado de color de piel en imágenes usando una combinación de espacios de colores HSV con YCbCr y HSV con CIELab. Se determina los intervalos de pixeles color no piel usando los componentes $\mathrm{H}$ y S, y los intervalos de pixeles color piel de los componentes $\mathrm{Cb}, \mathrm{Cr}$, a y b. Se compara los resultados de la técnica HS-CbCr que es la combinación de los componentes $\mathrm{H}$ y S (pixeles color no piel) con los componentes $\mathrm{Cb}$ y $\mathrm{Cr}$ (pixeles color piel), contra la técnica HSab que de la misma forma es la combinación de $\mathrm{H}$ y $\mathrm{S}$ pero con los componentes a y b (pixeles color piel). Se analiza la eficiencia de cada técnica para la segmentación de piel en base al porcentaje de detección de piel y no piel. Los resultados experimentales mostraron que la técnica HS-ab es mejor que la técnica HS-CbCr por la precisión además se descarta los obstáculos de luminancia pues en ambas técnicas se elimina la componente de luminancia.
\end{abstract}

Palabras clave: CIELab, crominancia, espacio de color, HSV, piel, YCbCr.

\section{Comparison of Two Proposed Techniques HS-CbCr and HS-ab for the Modeling of Skin Color in Images}

\begin{abstract}
The content of this paper is the proposal of two techniques for the modeling of skin color in images using a combination of spaces of colors HSV with $\mathrm{YCbCr}$ and $\mathrm{HSV}$ with CIELab. To determine the intervals of pixels nonskin color uses the components $\mathrm{H}$ and $\mathrm{S}$, on the other hand, the intervals of pixels skin color uses the components $\mathrm{Cb}, \mathrm{Cr}$, a and $\mathrm{b}$. There are compared the results of the technique HS-CbCr which is the combination of the components $\mathrm{H}$ and $\mathrm{S}$ (pixels non-skin color) with the components $\mathrm{Cb}$ and $\mathrm{Cr}$ (pixels skin color), against the technique HS-ab that of the same form is the combination of $\mathrm{H}$ and $\mathrm{S}$ but with the components a and $b$ (pixels skin color). It analyzes the efficiency of each technique for the segmentation of skin based on the percentage of detection of skin and non-skin. The experimental results showed that technique HS-ab is better than the technique HS-CbCr because of the precision in addition the obstacles of luminance are discarded because in both techniques the luminance component is eliminated.
\end{abstract}

Keywords: CIELab, chrominance, color space, HSV, skin, YCbCr 


\section{Introducción}

La detección de color de la piel humana en imágenes es una rama de investigación amplia en las áreas de visión por computador y procesamiento de imágenes. Algunas aplicaciones es el reconocimiento facial y/o corporal, vigilancia por video, interacción persona-ordenador (Human Computer Interaction-HCI), entre otras. Los problemas que se enfrentan para la detección de piel son el color, la orientación, la postura, la escala de la piel así como las condiciones de iluminación y los fondos complejos. La información de color es muy útil para extraer las regiones de piel en una imagen, además permite un procesamiento rápido y aporta robustez en la aplicación. De acuerdo a la literatura [1,2], los puntos esenciales para la detección de piel son la selección del espacio de color, el modelado de la distribución de color y depurar las regiones de la piel extraídas de la segmentación anterior.

En el primer punto, es de suma importancia la selección de espacio de color ya que determina la eficiencia del método para detectar el color de piel. Una desventaja que se presenta en el color es la sensibilidad al cambio de color de iluminación sobre todo en el espacio de color RGB. Una forma de resolver este problema es transformar la imagen RGB a otro espacio de color. Existe una variedad de espacio de colores para la clasificación de colores, en este caso nuestro interés es la segmentación de color de piel. La segmentación de color de piel determina si el pixel de color de una imagen es un color de piel o no es color de piel. Una buena segmentación de color de piel es aquella que segmenta cada color de piel ya sea negruzco, amarillento, pardo, blanquecino y aporta buenos resultados bajo las diferentes condiciones de luz. Los espacios de colores para el modelado de la piel se realizan mediante los componentes de crominancia porque se espera que la segmentación de color de piel pueda llegar a ser más resistente a las variaciones de iluminación si se descarta los componentes de luminancia [3]. En este documento los espacios de color $\mathrm{HSV}, \mathrm{YCrCb}$ y CIELab se utilizan para la segmentación de color de la piel debido a que separan los componentes de crominancia y luminancia logrando una caracterización de los diferentes colores de piel [4,5].

En el segundo punto, el modelado del color de la piel es construir una regla de decisión que discrimine o diferencie entre los píxeles de una imagen que corresponden al color de la piel y aquéllos que no. Esto puede llevarse a cabo por definiciones explícitas de regiones de color, modelado no paramétrico de la distribución de piel o modelado paramétrico de la distribución de piel [6].

En el tercer punto, después de la segmentación de color de piel en la imagen puede contener ruido y algunas imperfecciones para mejorar la imagen y eliminar el ruido se hace uso de las operaciones morfológicas [7].

En este artículo abarca los dos primeros puntos, en el primer punto como elemento primordial se elige el espacio de color en este caso se optó por tres espacios de colores HSV, YCrCb y CIELab; posteriormente para el modelado de color de piel se analizaron los histogramas de las imágenes para identificar los pixeles de color de piel y por último se realiza el algoritmo para detectar las regiones de piel en las imágenes. La aportación del artículo es la presentación de dos técnicas de modelos de color para la detección de color de piel. Ambas técnicas utilizan el espacio de color HSV que proporciona información adicional de tono y crominancia de una imagen con la finalidad de mejorar la discriminación entre los píxeles de la piel y píxeles no de piel [8]. En este trabajo el espacio de color HSV se usó para detectar los pixeles de color no piel posteriormente, 
en un método se aplica el espacio de color $\mathrm{YCbCr}$ para detectar los pixeles de color de piel y en el otro método se usa el espacio de color CIELab. Finalmente se realiza un análisis de histogramas de imágenes de piel de la base de datos SFA [9] para determinar el número de umbral de cada espacio de color que se implementa en el algoritmo.

\section{Espacio de color para el modelado de color de piel}

Se define espacio de color como una representación matemática de un conjunto de colores [10]. Hay una variedad de espacios de colores disponibles se dividen en cuatro grupos, algunos espacios de colores más usados se muestran en la Tabla 1.

Tabla 1. Grupos de espacios de colores más comunes

\begin{tabular}{ll}
\hline \multicolumn{1}{c}{ Grupo } & \multicolumn{1}{c}{ Espacio de colores } \\
\hline Modelo de color básico & RGB, RGB Normalizado, XYZ \\
Modelo de color perceptual & HIS, HSV, HSL, TSL, TSV \\
Modelo de color ortogonal & YCbCr, YIQ, YES, YUV \\
Colores perceptualmente uniforme & CIELab, CIELuv,CIE XYZ, CIE-xy Y \\
\hline
\end{tabular}

La mayor parte de la investigación en la detección de color de piel se basa en los espacios de color HIS, HSV, YCbCr y CIELab [3,11, 12]. A continuación se explicará brevemente los espacios de color HSV, YCbCr y CIELab.

\subsection{HSV}

El problema del espacio de color RGB (en inglés Red, Green and Blue) no proporciona la información correcta sobre el color de piel debido a los efectos de luminancia. HSV ofrece información de tono (en inglés Hue) que define el color dominante (como el rojo, verde, morado y amarillo) de una zona y tiene un rango de $0^{\circ}$ a $360^{\circ}$, saturación (en inglés Saturation) que mide el colorido de un área en proporción a su brillo y tiene un rango de $0 \%$ a $100 \%$, y valor (en inglés Value) se refiere al brillo del color y provee una noción acromática de la intensidad de color. Los componentes $\mathrm{H}$ y $\mathrm{S}$ entregan información útil para la discriminación en la búsqueda de piel, es por eso que se emplea para detectar a los pixeles de color no piel [13]. Para convertir el espacio de color RGB al HSV se usan las siguientes expresiones matemáticas:

$$
\begin{gathered}
H=\arccos \frac{1 / 2[(R-G)+(R-B)]}{\sqrt{\left[(R-G)^{2}+(R-B)(G-B)\right]}}, \\
S=1-3 \frac{\mathrm{min}(R, G, B)}{R+G+B}, \\
V=\frac{1}{3}(R+G+B),
\end{gathered}
$$

donde $\mathrm{H}$ es tono, $\mathrm{S}$ es saturación, $\mathrm{V}$ es valor, $\mathrm{R}$ es el componente rojo, $\mathrm{G}$ es el componente verde y B es el componente azul. En la Fig. 1, se muestra el modelo HSV 
donde su sistema coordenado es cilíndrico y su subespacio es una pirámide de base hexagonal.
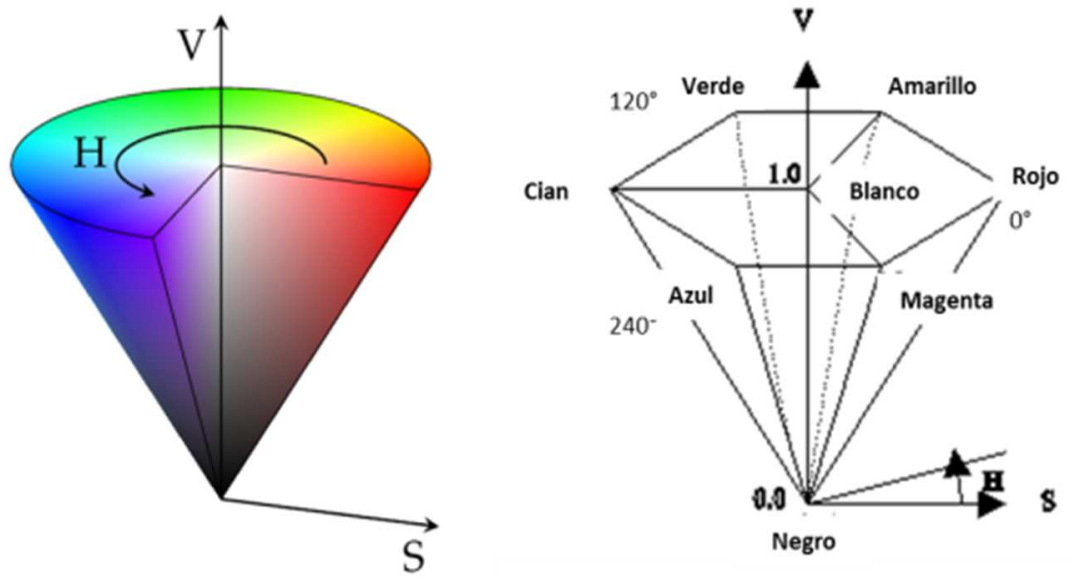

Fig. 1. Modelo HSV. La tonalidad se representa por valores del grado de un ángulo $\left(0^{\circ}-360^{\circ}\right)$, la saturación se representa como la distancia del eje del brillo (negro-blanco) y el valor representa la altura en el eje (negro-blanco)

\section{2. $\mathrm{YCbCr}$}

En este espacio de color la información de la luminancia está representada por una solo componente Y, por otro lado la información de color es almacenada en los componentes $\mathrm{Cb}$ y $\mathrm{Cr}$. $\mathrm{Cb}$ es la componente de crominancia azul y se define como la diferencia entre la componente azul y el valor de referencia ver ecuación (5). Cr es la componente de crominancia roja y como se ve en la ecuación (6) es la diferencia entre la componente roja y el valor de referencia [14]. Para transformar el espacio de color RGB a YCbCr se lleva acabo con las siguientes ecuaciones:

$$
\begin{gathered}
Y=0.299 R+0.587 G+0.114 B, \\
C b=B-Y, \\
C r=R-Y,
\end{gathered}
$$

donde $\mathrm{R}$ es el componente rojo, $\mathrm{G}$ es el componente verde y B es el componente azul. La Fig. 2 expone una representación gráfica del espacio de color YCbCr.

\subsection{CIELab}

Es un modelo cromático para describir todos los colores que puede percibir el ojo humano. C.I.E. (Commission Internationale d'Eclairage) es un organismo encargado de especificar los estándares de color como CIEXYZ, CIELuv, y CIELab. La componente $\mathrm{L}$ es la luminancia (va de negro a blanco) y los componentes a,b son 
colores cromáticos (en a oscila entre rojo a verde mientras en b oscila entre azul a amarillo) [15]. El modelo CIELab se modela en la Fig. 3.

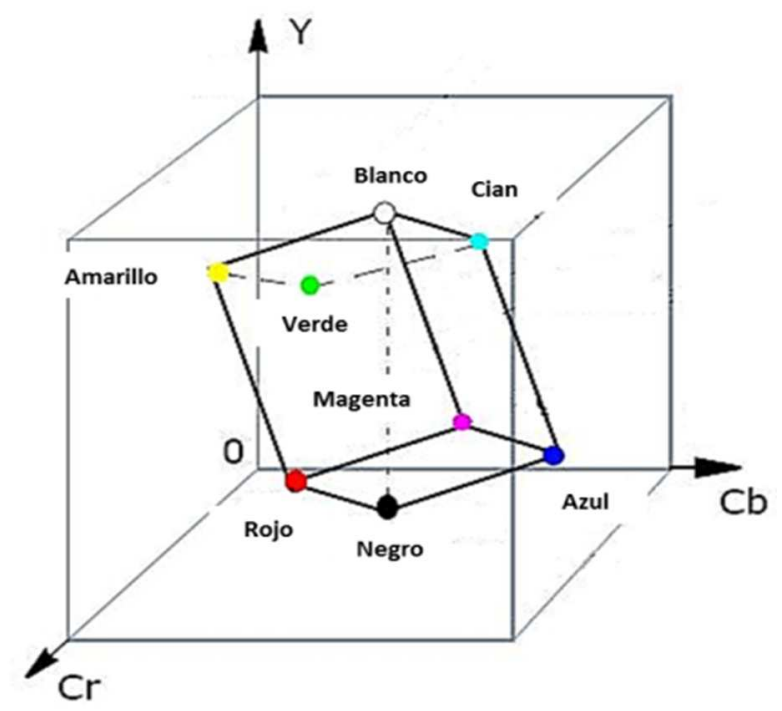

Fig. 2. Modelo YCbCr. Todos los colores posibles de RGB ocupan sólo una parte del espacio de color $\mathrm{YCbCr}$ (cubo interior). En el cubo grande representa todos los valores posibles de $\mathrm{YCbCr}$

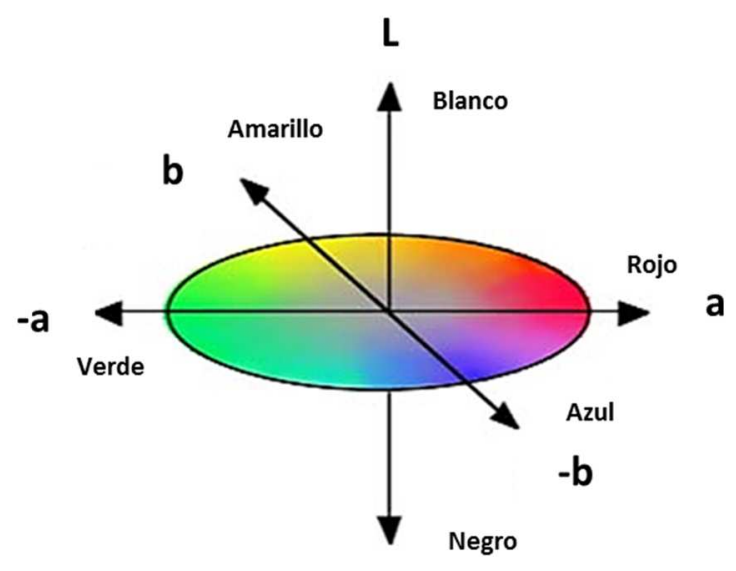

Fig. 3. Modelo CIELab. $\mathrm{L}=0$ proporciona el color negro y $\mathrm{L}=100$ proporciona el color blanco. Los valores $-\mathrm{a}<0$ indican verde mientras que los valores $\mathrm{a}>0$ indican rojo. Los valores $\mathrm{de}-\mathrm{b}<0$ indican azul y los valores de $b>0$ indican amarillo

\section{Metodología}

El procedimiento para el desarrollo de este trabajo es el siguiente: 


\subsection{Cálculo de umbral}

Se obtienen 40 imágenes de diferentes colores y texturas, y se descargan 40 imágenes de la base de datos SFA que contiene una variedad de colores de piel. En el primer caso, cada imagen se transforma del modelo de color RGB a HSV obteniendo los histogramas de los componentes H y S. En dichos histogramas se tienen los valores máximos y mínimos de los distintos tonos de piel de cada componente. Con todos los valores que se obtuvieron de las 40 imágenes se tiene el valor promedio para finalmente tener el intervalo del componente $\mathrm{H}$ y $\mathrm{S}$. Para el segundo caso es el mismo procedimiento excepto que las imágenes se transforman al modelo de color YCbCr y CIELab; por lo tanto se tienen los intervalos de los componentes $\mathrm{Cb}, \mathrm{Cr}$, a y b. El procedimiento se simplifica en la Fig. 4.
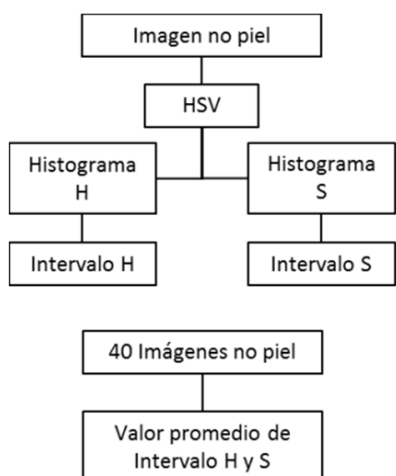
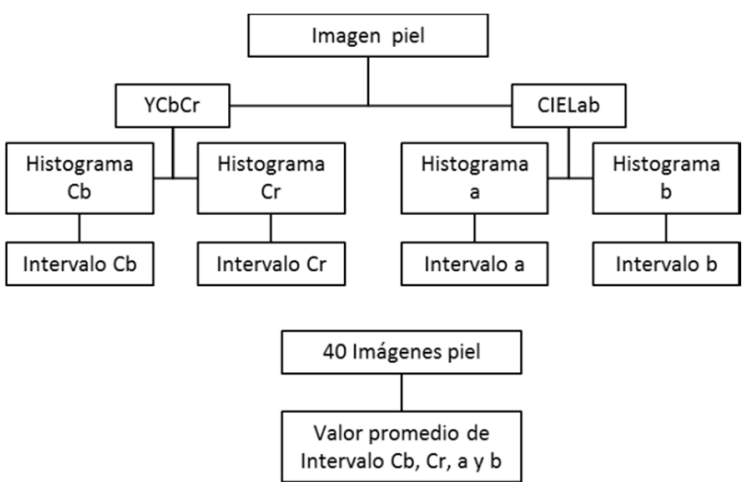

Fig. 4. Diagrama para cálculo de umbral. En la parte de arriba se describe el proceso de una sola imagen tanto de la no piel como la de piel. En la parte de abajo indica que después de obtener los intervalos de las 40 imágenes se obtiene el intervalo promedio

\subsection{Técnicas HS-CbCr y HS-ab}

Después de conocer el umbral de los intervalos de cada espacio de color. Se desarrolla un algoritmo que nos va a permitir detectar las regiones de piel de una imagen. Este algoritmo se implementa en MATLAB (R2015b-The MathWorks). Este algoritmo consiste en cargar la imagen al programa, transformar la imagen en espacio de color HSV e identificar los pixeles no piel con los intervalos $\mathrm{H}$ y S para descartarlos; después con esa misma imagen se convierte al espacio de color YCbCr para seleccionar los pixeles de color piel con el intervalo $\mathrm{Cb}$ y $\mathrm{Cr}$ y se despliega la imagen con las regiones de solo piel, esta es la técnica HS-CbCr. Por otro lado la técnica HS-ab tiene el mismo procedimiento descrito anteriormente a excepción que para identificar los pixeles de color de piel es con el intervalo a y b. Por último en ambas técnicas, se calcula el porcentaje de piel de la imagen contando todos los pixeles que sean diferentes de cero o diferentes de negro, es decir, la zona más grande de piel en la imagen (A) entre el número total de pixeles de color de piel (B). Esto se define en la ecuación (7).

$$
\% \text { piel }=\frac{A}{B} * 100 \% \text {. }
$$


En la Fig. 5, se presenta el diagrama de flujo del algoritmo descrito previamente.

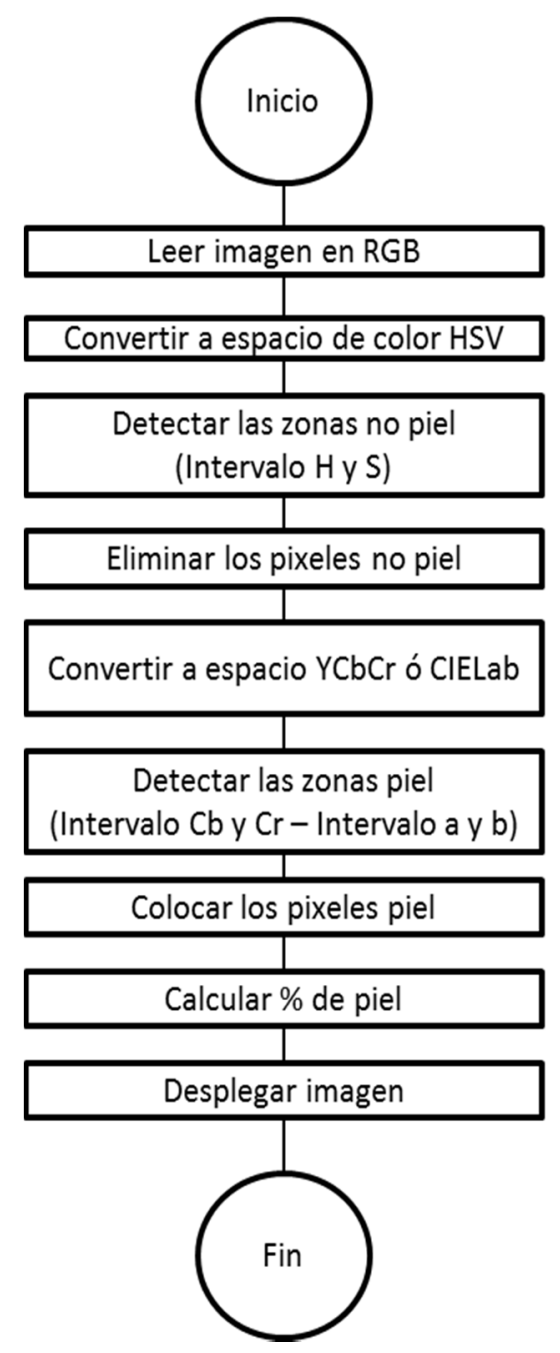

Fig. 5. Algoritmo de las técnicas HS-CbCr y HS-ab. Como se observa es el mismo algoritmo para las dos técnicas sólo cambia la conversión de modelo de color YCbCr y CIELab con sus respectivos intervalos

\subsection{Evaluación}

Después La evaluación se realiza con el fin de determinar qué componentes de crominancia producen los mejores resultados de la detección de piel. Esta evaluación consiste en obtener el número de pixeles que son clasificados de color de piel tanto para el espacio de color YCbCr y CIELab de las 40 imágenes de piel, también se obtiene el número total de pixeles de las imágenes. Esta evaluación está expresada en la ecuación (8): 
Diana Alejandra Contreras Alejo, Francisco Javier Gallegos Funes

$$
\% C=\frac{\text { No.pixelesdecolorpiel }}{\text { No.pixelestotal }} * 100 \% \text {. }
$$

\section{Resultados}

Las 40 imágenes color no piel y 40 imágenes color piel que se usaron se presenta en la Fig. 6. Cada imagen se transformó al espacio de color HSV y se obtuvo su histograma de las canales $\mathrm{H}$ y $\mathrm{S}$ para obtener los valores mínimos y máximos de cada canal. Este proceso se repite para el espacio de color YCbCr y para el espacio de color CIELab, un ejemplo se observa en la Fig. 7 donde se convierte una imagen de color piel en el espacio de color YCbCr presentando sus respectivos histogramas de cada canal. En este caso el intervalo es $\mathrm{Cb}=[110,114]$ y $\mathrm{Cr}=[138,142]$. Al tener todos los valores mínimos y máximos de cada espacio de color, se tiene el valor promedio para tener el intervalo de las imágenes no piel en modelo HSV: $0<\mathrm{H}<0.2$ y $0.15<\mathrm{S}<0.9$, el intervalo de las imágenes piel en modelo YCbCr: $88<\mathrm{Cb}<130$ y $127<\mathrm{Cr}<175$, y el intervalo de las imágenes piel en modelo CIELab: $142<$ a $<225$ y $115<$ b $<177$.

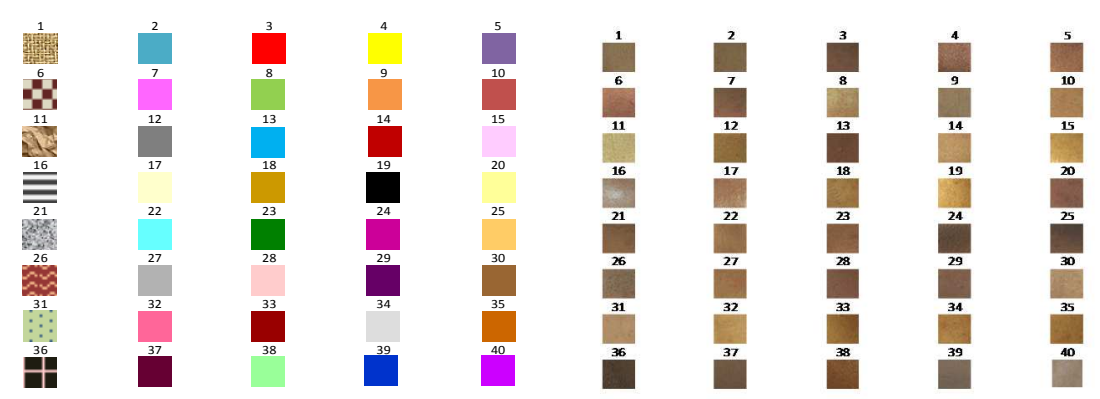

Fig. 6. Imágenes. Las imágenes color no piel son aquellas imágenes con una variedad de colores y texturas mientras las imágenes color piel son imágenes reales de diversos tipos de piel
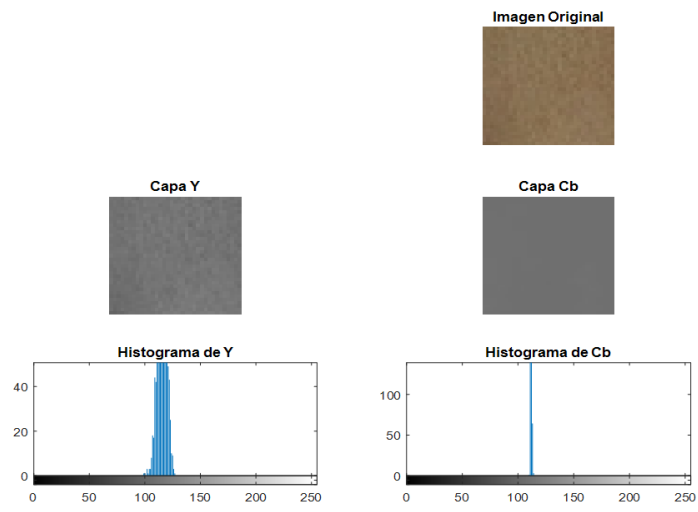

Fig. 7. Histogramas de las capas de color $\mathrm{YCbCr}$ en una imagen de piel. La imagen original en RGB se convierte en espacio de color $\mathrm{YCbCr}$, y se tiene el histograma de $\mathrm{Y}, \mathrm{Cb}$ y $\mathrm{Cr}$; en este caso los valores que nos interesan son los de componentes de crominancia es decir $\mathrm{Cb}$ y $\mathrm{Cr}$ 
Los intervalos se implementaron en el algoritmo, se usaron 20 imágenes reales de personas para la detección de piel. En la Tabla 2, se expone los resultados de los porcentajes de piel de cada técnica. Y se observa una gran diferencia en los porcentajes de detección de piel de la técnica $\mathrm{HS}-\mathrm{CbCr}$ con respecto a la técnica $\mathrm{HS}$-ab, entonces se podría decir que al comparar ambas técnicas, la primera detecta mayor cantidad de regiones de piel en la imagen.

Tabla 2. Porcentaje de detección de piel y no piel de la técnica HS-CbCr y de la técnica HS-ab

\begin{tabular}{cccc}
\hline No. & $\begin{array}{c}\text { Técnica HS-CbCr } \\
\text { \% de piel }\end{array}$ & $\begin{array}{c}\text { Técnica HS-ab } \\
\text { \% de piel }\end{array}$ & \% de no piel \\
\hline 1 & 28.31 & 11.87 & 29.25 \\
2 & 52.88 & 4.19 & 52.89 \\
3 & 27.42 & 4.29 & 27.69 \\
4 & 34.44 & 16.68 & 36.22 \\
5 & 59.88 & 21.67 & 59.99 \\
6 & 53.57 & 10.80 & 53.67 \\
7 & 51.31 & 8.56 & 51.57 \\
8 & 8.49 & 0.54 & 8.77 \\
9 & 39.79 & 4.60 & 41.07 \\
10 & 9.26 & 4.31 & 9.27 \\
11 & 23.14 & 5.03 & 25.61 \\
12 & 32.94 & 5.70 & 34.19 \\
13 & 21.42 & 5.42 & 21.55 \\
14 & 74.97 & 10.14 & 75.35 \\
15 & 44.13 & 35.17 & 44.44 \\
16 & 69.31 & 6.24 & 70.19 \\
17 & 13.07 & 1.62 & 13.30 \\
18 & 63.79 & 30.03 & 63.96 \\
19 & 39.14 & 28.86 & 39.90 \\
20 & 31.97 & 4.20 & 32.30 \\
\hline
\end{tabular}

Sin embargo en la Fig. 8, se muestran cinco imágenes ya procesadas con ambas técnicas; y a simple vista la mejor detección de piel es en la técnica HS-ab ya que detecta sola la piel mientras en la técnica HS-CbCr presenta falsas alarmas pues detecta la piel y otros factores (fondo, cabello, etc.) que presentan colores similares a la piel.

Tabla 3. Porcentaje de detección de piel en los componentes de crominancia

\begin{tabular}{ccc}
\hline Espacio de color & Crominancia & \% C \\
\hline YCbCr & $\mathrm{CbCr}$ & 33.51 \\
$\mathrm{CIELab}$ & $\mathrm{ab}$ & 34.80 \\
\hline
\end{tabular}


Para evaluar cuantitativamente que espacio de color detecta más preciso el color de piel se aplica la ecuación (8). En la Tabla 3 se presenta los porcentajes de detección de piel en los componentes de crominancia; y se concluye que el espacio de color CIELab es más preciso en la detección de piel con respecto a YCbCr.
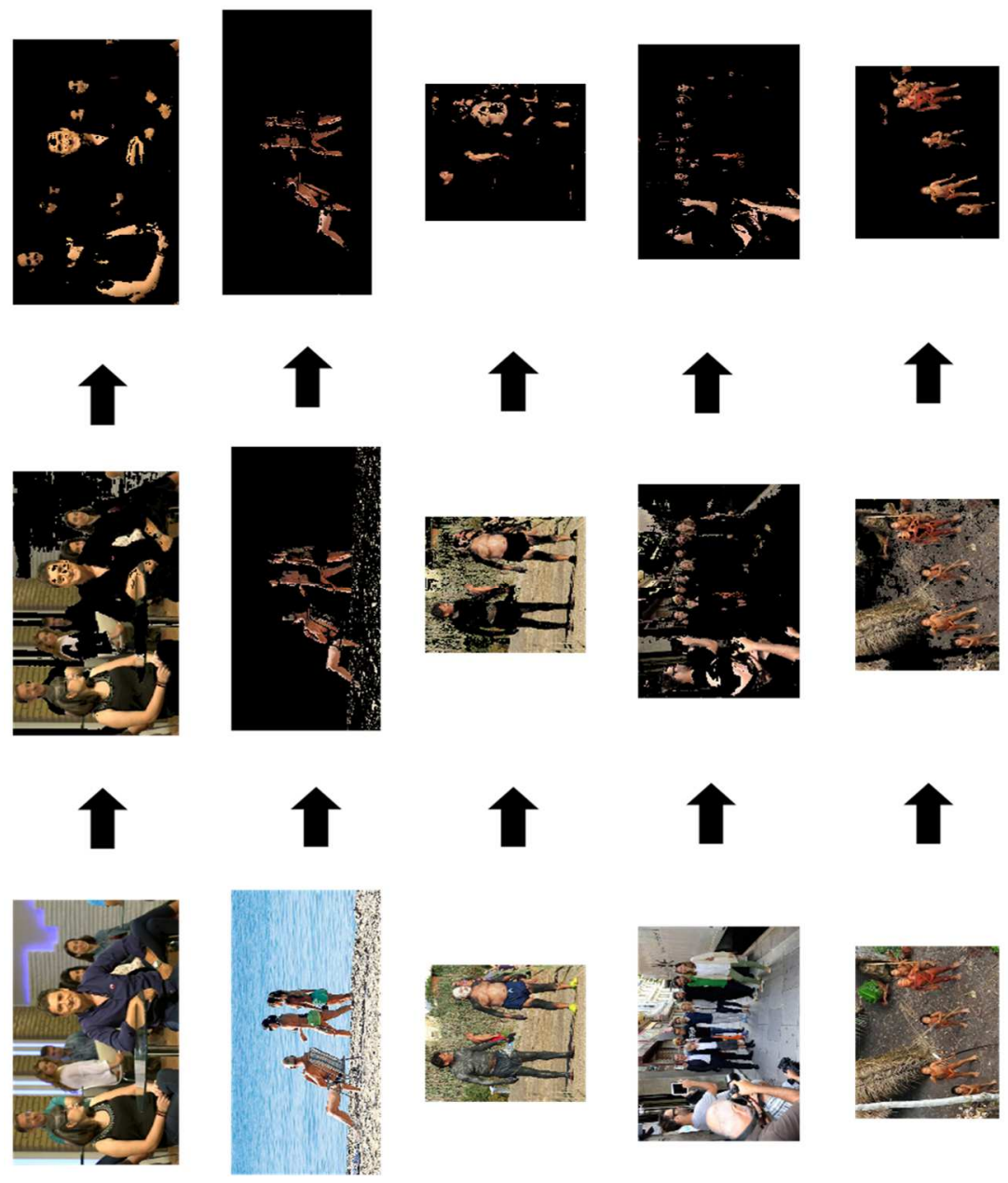

Fig. 8. Imágenes con aplicación de las técnicas propuestas. De izquierda a derecha, en la primera columna se muestran las imágenes reales (originales, en la segunda columna son las imágenes con la aplicación de la técnica HS-CbCr, y en la última las imágenes son procesadas con la técnica HS-ab

\section{Conclusiones}

Hoy en día, el avance de investigación en los métodos de detección de piel es necesario en múltiples disciplinas. Una técnica apropiada para detectar los pixeles de 
Comparación de dos técnicas propuestas HS-CbCr y HS-ab para el modelado de color de piel ...

color de piel y no color de piel es vital para imágenes con piel de diferente tipos de piel como el rosa, amarillo, blanco, marrón oscuro y marrón claro. En este trabajo, se presenta la innovación y comparación de dos técnicas para el modelado de color de piel. La segmentación de regiones de piel fue desarrollada usando los intervalos $\mathrm{H}$ y $\mathrm{S}$ de no piel del modelo de color HSV con el espacio de color YCbCr y CIELab del cual en ambas técnicas demuestra una clara discriminación entre regiones de piel y no piel. Los resultados experimentales mostraron que nuestro nuevo enfoque de modelado de color de piel fue capaz de lograr la detección de piel con éxito en todas las imágenes. De acuerdo con los resultados, en la primera técnica (HS-CbCr) se presenta un mayor porcentaje de detección de piel comparado con la segunda técnica (HS-ab) pero eso no indica que es mejor la primera técnica; ya que en todos de los casos se presentó una diferencia mínima respecto al porcentaje de pixeles no piel con el porcentaje de detección de piel de $\mathrm{HS}-\mathrm{CbCr}$; eso significa que la técnica $\mathrm{HS}-\mathrm{CbCr}$ presenta una confusión de identificar el color por ejemplo detecta los colores del cabello castaño, güero como color de piel. Sin embargo en la técnica HS-ab su porcentaje de detección de color de piel es mucho menor que el porcentaje de pixeles no piel además se observa que descarta los colores de cabello u otros parecidos al de la piel y el resultado de \%C del espacio de color CIELab fue mayor que YCbCr eso indica que los componentes de crominancia en el primer espacio de color son más precisos en la detección de color de piel. Por lo tanto se concluye que es fiable la segunda técnica HS-ab para la detección de color de piel además se reafirma lo mencionado en la literatura [3] con respecto a que la segmentación del espacio de color CIELab es mejor que YCbCr que da más información de color que otros espacio de colores por lo que es más preciso.

Agradecimientos. Los autores agradecen al Instituto Politécnico Nacional de México (IPN) y al Consejo Nacional de Ciencia y Tecnología de México (CONACYT) por la ayuda y el apoyo para desarrollar la Investigación. Como la colaboración y apoyo otorgado por parte de los Doctores del posgrado de la S.E.P.I. de la Escuela Superior de Ingeniería Mecánica y Eléctrica Unidad Profesional Zacatenco.

\section{Referencias}

1. Vezhnevets, V., Sazonov V., Andreeva, A.: A survey on pixel-based skin color detection techniques. In: GRAPHICON'03, pp. 85-92 (2003)

2. Prema, C., Manimegalai, D.: Survey on Skin Tone Detection using Color Spaces. International Journal of Applied Information Systems, Published by Foundation of Computer Science, New York, USA, Vol. 2, No. 2, pp. 18-26, (2012)

3. Amanpreet, K., Kranthi, B.: Comparison between YCbCr Color Space and CIELab Color Space for Skin Color Segmentation. International Journal of Applied Information Systems Published by Foundation of Computer Science, New York, USA, Vol. 3, No. 4, pp. 30-33, July (2012)

4. Phung, S.L., Bouzerdoum, A., Chai, D.: Skin segmentation using color pixel classification: analysis and comparison. IEEE Transactions on Pattern Analysis and Machine Intelligence, Vol. 27, No. 1 (2005)

5. Sabottka, K., Pitas, I.: Segmentation and Tracking of Faces in Color Images. In: AFGR'96, Killington, Vermont, pp. 236-241 (1996) 
6. Aznaveh, M.M., Mirzaei, H., Roshan, E., Saraee, M.: A new color based method for skin detection using RGB vector space. In: Human System Interactions, Conference on, Krakow, pp. 932-935 (2008)

7. Bhata, V.S., Pujaria, J.D.: Face detection system using HSV color model and morphing operations. International Journal of Current Engineering and Technology, pp. 200204 (2013)

8. Tabassum, M.R., Gias, A.U., Kamal, M.M., Muctadir, H.M., Ibrahim, M., Shakir, A.K., Imran, A., Islamm, S., Rabbani, G., Khaled, S.M., Islam, S., Begum, Z.: Comparative Study of Statistical Skin Detection Algorithms for Sub-Continental Human Images. Information Technology Journal, Vol. 9, No. 4, pp. 811-817 (2010)

9. Casati, J.P.B., Moraes, D.R., Rodrigues, E.L.L.: SFA: A Human Skin Image Database based on FERET and AR Facial Images. In: IX Workshop de Vision Computational (2013)

10. Fairchild, M.D.: Color Appearance Models. John Wiley \& Sons. ed.3, p. 472 (2013)

11. Lee, Y.J., Yoo, S.I.: An elliptical boundary model for skin color detection. In: International Conference on Imaging Science, Systems, and Technology, pp. 24-27 (2002)

12. Chai, D., Bouzerdoum, A.: A Bayesian approach to skin color classification in YCbCr color space. In: Institute of Electrical and Electronics Engineers IEEE Region Ten Conference (TENCON), Vol. 2, pp. 421-424 (2000)

13. Qiong, L., Guang-zheng, P.: A robust skin color based face detection algorithm. In: Informatics in Control, Automation and Robotics (CAR), 2nd International Asia Conference on, Wuhan, pp. 525-528 (2010)

14. Phung, S.L., Bouzerdoum, A., Chai, D.: A novel skin color model in YCbCr color space and its application to human face detection. In: Image Processing Proceedings International Conference on, Vol. 1, pp. I-289-I-292 (2002)

15. Sharma, G., Bala, R.: Digital Color Imaging Handbook: Electrical Engineering \& Applied Signal Processing Serie. CRC Press, pp. 32-45 (2002) 